\title{
Hipóxia neonatal e ocorrência do diagnóstico de epilepsia na infância: uma revisão sistemática
}

\author{
Neonatal hypoxia and occurrence of the diagnosis of epilepsy in childhood: a systematic \\ review
}

Hipóxia neonatal y ocurrencia del diagnóstico de epilepsia en la infancia: una revisión sistemática

Jéssica Nayara Pereira Jatobá1*, Carla Magda de Morais Cardoso², Tatiane Pereira Horta ${ }^{2}$, Franciele Evangelista Silva², Matheus Felipe de Oliveira Rocha ${ }^{2}$, Débora Soares Cardoso², Luana Mendes de Castro², Anna Caroline Souza ${ }^{2}$, Camila Oliveira Fonseca², Jéssica Gonçalves Porto², Cinthia Moreira de Araújo Melo², Smith Evangelista Caldeira², Mariana de Souza Guedes², Patrícia Costa Gomes², Renê Ferreira da Silva Júnior¹.

\section{RESUMO}

Objetivo: Descrever qual a relação entre hipóxia neonatal e ocorrência de epilepsia na infância por meio de uma revisão sistemática da literatura. Métodos: Estudo com característica exploratória realizada através da metodologia de uma revisão sistemática de literatura. O cenário da pesquisa foi a rede mundial de computadores (wordwide web) utilizando como fonte de pesquisa as bases de dados Pubmed, Medline, Lilacs e Scielo, selecionando artigos científicos de acordo com os critérios PRISMA (2009) que referem a legitimidade através do PICOS (População/doença, Intervenção/exposição de interesse, Comparador, Outcomes/principal desfecho, Study design). A consulta as bases de dados ocorreram em abril de 2018, sem restrição de ano de publicação. Resultados: Selecionamos pela triagem 29 artigos para a leitura do texto completo. Dentre esses incluiu nove artigos no estudo, destes todos falavam que a hipóxia tinha relação com a epilepsia. Considerações finais: Diante do presente estudo pode se verificar que hipóxia neonatal é uma das causas da epilepsia. Sugerimos novos estudos diante da escassez de artigos sobre o tema.

Palavras-chave: Epilepsia, Parto, Criança, Hipóxia.

\begin{abstract}
Objective: To describe the relationship between neonatal hypoxia and the occurrence of epilepsy in childhood through a systematic review of the literature. Methods: This is an exploratory study carried out using the methodology of a systematic literature review. The search scenario was the world wide web using the Pubmed, Medline, Lilacs and Scielo databases as the research source, selecting scientific articles according to PRISMA criteria (2009) that refer to legitimacy through PICOS (Population / disease, Intervention / exposure of interest, Comparator, Outcomes / main outcome, Study design). The query to the databases occurred in April 2018, without restriction of year of publication. Results: We selected 29 articles for the reading of the full text. Among these included nine articles in the study, of which, all said that hypoxia was related to epilepsy. Final considetarions: In the present study it can be verified that neonatal hypoxia is one of the causes of epilepsy. We suggest new studies in the face of the scarcity of articles on the subject.
\end{abstract}

Key words: Epilepsy, Childbirth, Child, Hypoxia.

${ }^{1}$ Faculdades Unidas do Norte de Minas - FUNORTE, Montes Claros-MG. *E-mail: nayarajessica23@yahoo.com.br ${ }^{2}$ Faculdades de Saúde Ibituruna - FASI. Montes Claros-MG.

SUBMETIDO EM: 12/2018

ACEITO EM: 1/2019

PUBLICADO EM: 6/2019

REAS/EJCH | Vol. Sup. 24 | e1136 | DOI: https://doi.org/10.25248/reas.e1136.2019 Página 1 de 6 


\section{RESUMEN}

Objetivo: Describir cuál es la relación entre hipoxia neonatal y ocurrencia de epilepsia en la infancia por medio de una revisión sistemática de la literatura. Métodos: Estudio con característica exploratoria realizada a través de la metodología de una revisión sistemática de literatura. El escenario de la investigación fue la red mundial de computadoras (wordwide web) utilizando como fuente de investigación las bases de datos Pubmed, Medline, Lilacs y Scielo, seleccionando artículos científicos de acuerdo con los criterios PRISMA (2009) que refieren la legitimidad a través del PICOS (Población / enfermedad, Intervención / exposición de interés, Comparador, Outcomes / principal desenlace, Estudio de estudio). La consulta de las bases de datos ocurrió en abril de 2018, sin restricción de año de publicación. Resultados: Seleccionamos por la clasificación 29 artículos para la lectura del texto completo. Entre estos incluyeron nueve artículos en el estudio, de éstos, todos hablaban que la hipoxia tenía relación con la epilepsia. Consideraciones finales:En el presente estudio se puede verificar que la hipoxia neonatal es una de las causas de la epilepsia. Sugerimos nuevos estudios ante la escasez de artículos sobre el tema.

Palabras clave: Epilepsia, Parto, Niño, Hipoxia.

\section{INTRODUÇÃO}

Atualmente, se sabe que a epilepsia é uma doença neurológica caracterizada por crises epiléticas, que provoca disfunção de um conjunto de neurônios (FERNANDES MJS, 2013). Nos primeiros anos de vida a epilepsia está diretamente relacionada com prematuridade e hipóxia ao nascer. Mesmo com os avanços tecnológicos da saúde ainda não se sanou esse problema relacionado ao parto, o que seria um grande avanço já que causas neurológicas são responsáveis por uma boa parte da morbidade de recém-nascidos. Um estudo feito com indivíduos que possuem síndrome de West que é uma epilepsia da infância e de difícil controle mostrou que mais de $70 \%$ dos pacientes do estudo tiveram hipóxia durante o parto ou eram prematuros, com isso pode se observar que um parto laborioso tem uma grande relação com o surgimento da epilepsia (MINAMIHARA CT et al., 2015).

Foram observados vários problemas neurológicos relacionados à hipóxia neonatal, entre eles estavam à diminuição do déficit cognitivo e os distúrbios epiléticos. Isso ocorre porque a falta de oxigênio no cérebro leva a morte dos neurônios deixando assim os neonatos susceptíveis a epilepsia (VELÁSQUEZ NH et al., 2011). Outro problema é a asfixia durante o parto que pode causar danos severos ao sistema neurológico do neonato, podendo levar principalmente ao surgimento da epilepsia. Inicialmente pode haver um mascaramento dos danos que com o passar dos meses aparecera de forma progressiva com complicações neurológicas graves (MINAMIHARA CT et al., 2015).

Pode se observar que uma das causas da epilepsia seria relacionada ao pré-natal e a diminuição ou falta de oxigênio durante o parto. Mas também se observou que a assistência durante o parto tanto à mãe quanto ao recém-nascido é um fator decisivo, já que uma má assistência pode gerar danos cerebrais e consequentemente epilepsia. A realização do pré-natal e um parto dentro do tempo ideal e com cautela para evitar lesões, poderia diminuir o número de crianças com epilepsia. O cuidado é um ponto essencial que não pode ser desprezado, até porque essa criança pode ter que conviver com uma doença a vida toda, e algumas crises de difícil controle, tomando medicações diariamente e muitas vezes sofrendo preconceitos (FONSECA GS et al., 2017). Sendo assim, o objetivo deste estudo é descrever qual a relação entre hipóxia neonatal e ocorrência de epilepsia na infância por meio de uma revisão sistemática da literatura.

\section{MÉTODOS}

Estudo com característica exploratória realizada através da metodologia de uma revisão sistemática de literatura. A revisão sistemática é um tipo de metodologia que trata de questões bem definidas, claras e objetivas visando a identificação, seleção e avaliação de artigos relevantes e por fim, síntese de questões evidentes para a literatura científica (GALVÃO TF e PEREIRA MG, 2013).O cenário da pesquisa foi a rede mundial de computadores (wordwide web) utilizando como fonte de pesquisa as bases de dados Pubmed, Medline, Lilacs e Scielo. 
Os critérios de inclusão utilizados foram artigos encontrados nas bases de dados Pubmed, Medline, Lilacs, Scielo e artigos relacionados a nossa pergunta de pesquisa. Os critérios de exclusão foram artigos de revisão e artigos que não fossem da língua inglesa, portuguesa ou espanhola. A consulta as bases de dados ocorreram em abril de 2018, sem restrição de ano de publicação. Os artigos foram lidos e selecionados após uma análise estatística de dados sobre a ocorrência de epilepsia infantil relacionada ao parto laborioso citados nos estudos. Foram selecionando artigos científicos de acordo com os critérios PRISMA (2009) que referem a legitimidade através do PICOS (População/doença, Intervenção/exposição de interesse, Comparador, Outcomes/principal desfecho, Study design). O PRISMA é uma recomendação para estudo de revisão sistemática de literatura que consiste em um checklist de 27 itens e um fluxograma que possui quatro etapas. Ele foi criado para auxiliar os pesquisadores no desenvolvimento da sua pesquisa, pode ser usado também para melhorar relatos de revisões já publicadas além de ser um método de avaliação de artigos (GALVÃO TF et al., 2015). Tivemos como limitação a linguagem estrangeira. Com isso não foram utilizados artigos em que não fossem da língua portuguesa, inglesa ou espanhola.

\section{RESULTADOS}

Identificou-se um total de 1564 artigos nas bases de dados consultadas. Foram removidos artigos por serem duplicatas, com isso realizou a leitura por título e resumo de 1043 estudos. Selecionamos pela triagem 29 artigos para a leitura do texto completo. Dentre esses incluiu nove artigos no estudo, destes todos falavam que a hipóxia tinha relação com a epilepsia (Figura 1).

Figura 1 - Fluxograma de seleção dos artigos para a revisão sistemática.

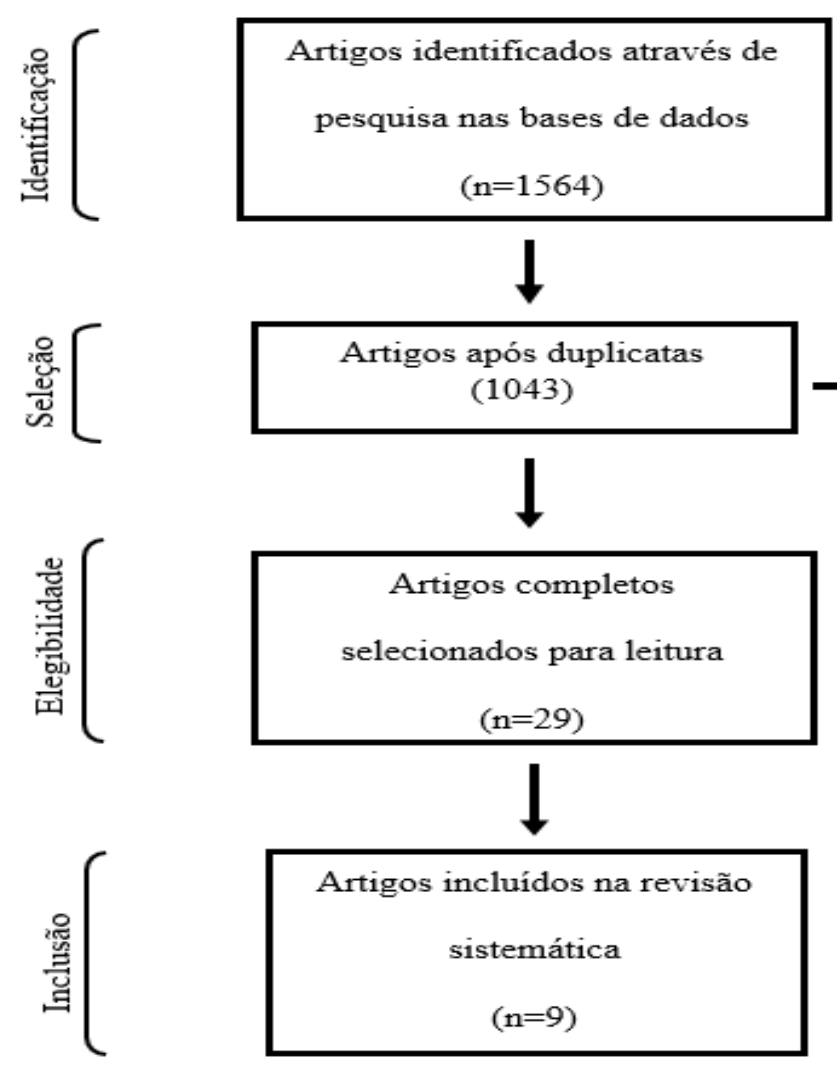

Fonte: Dados da pesquisa, 2018.

Os estudos foram de diferentes países, entre eles Turquia (1), Brasil (2), Estados Unidos da América-EUA (1), Itália (2), Cuba (2) e Índia (1). O Ano de publicação dos estudos foram de 1997 a 2017, sendo que um artigo foi publicado na década de 90 e os outros oito a partir de 2006 (Quadro 1). 
Quadro 1 - Quadro sinóptico.

\begin{tabular}{|c|c|c|c|c|}
\hline Autor, ano, local & Título do artigo & Método & $\mathbf{N}$ & $\begin{array}{l}\text { Principais resultados } \\
\end{array}$ \\
\hline $\begin{array}{l}\text { KUMAR et al., } 2015 \\
\text { (IIndia) }\end{array}$ & $\begin{array}{l}\text { Relação da síndrome de } \\
\text { Lennox-Gastaut com evento } \\
\text { perinatal: estudo transversal. }\end{array}$ & $\begin{array}{l}\text { Estudo } \\
\text { transversal }\end{array}$ & 31 & $\begin{array}{l}\text { No grupo de eventos perinatais, } 10 \text { das crianças tinham hipóxia perinatal, cinco } \\
\text { tinham septicemia e duas tinham hipoglicemia. Desde então, uma grande proporção } \\
\text { de crianças com LGS sofre de hipóxia perinatal; classificar o LGS em grupos com e } \\
\text { sem o evento perinatal é útil para o clínico na previsão do prognóstico. }\end{array}$ \\
\hline $\begin{array}{l}\text { ÁLVAREZ; MARTÍNEZ, } \\
2012 \\
\text { (Cuba) }\end{array}$ & $\begin{array}{l}\text { Caracterização etiológica da } \\
\text { síndrome de Lennox-Gastaut } \\
\text { sintomática. }\end{array}$ & $\begin{array}{l}\text { Estudo descritivo, } \\
\text { retrospectivo }\end{array}$ & 36 & $\begin{array}{l}\text { Pode-se concluir que as etiologias mais frequentes do SLG em nosso meio são as } \\
\text { malformações congênitas do sistema nervoso central (SNC) e a hipóxia perinatal. }\end{array}$ \\
\hline $\begin{array}{l}\text { ARGUDÍN et al., } 2011 \\
\text { (Cuba) }\end{array}$ & $\begin{array}{l}\text { Estudo epidemiológico da } \\
\text { epilepsia infantil no município de } \\
\text { Bahía Honda. }\end{array}$ & $\begin{array}{l}\text { Estudo } \\
\text { observacional, } \\
\text { descritivo e } \\
\text { transversal. }\end{array}$ & 43 & $\begin{array}{l}\text { Ao analisar essa variável de forma particular, observou-se que a associação entre } \\
\text { hipóxia (12,5\%), prematuridade e baixo peso ao nascer (10,0\%), respectivamente, } \\
\text { predominou entre os fatores perinatais; Infecções do sistema nervoso Central (SNC) } \\
\text { (10,0\%) nos fatores pós-natal e misto foi mais frequente a associação entre esses } \\
\text { fatores. }\end{array}$ \\
\hline $\begin{array}{l}\text { PISANI et al., } 2009 \\
\text { (Itália) }\end{array}$ & $\begin{array}{l}\text { Desenvolvimento de epilepsia em } \\
\text { recém-nascidos com encefalopatia } \\
\text { hipóxico-isquêmica moderada e } \\
\text { convulsões neonatais. }\end{array}$ & Ensaio Cínico & 92 & $\begin{array}{l}\text { Dos } 92 \text { recém-nascidos com asfixia perinatal, apenas } 57 \text { os sujeitos desenvolveram } \\
\text { HIE mais tarde, } 41 \text { machos e } 16 \text { fêmeas. Apenas } 18 \text { indivíduos }(31,6 \%) \\
\text { apresentaram crises neonatais como consequência da EH revelou que apenas a } \\
\text { variável HIE estava relacionado ao desenvolvimento de epilepsia pós neonatais. }\end{array}$ \\
\hline $\begin{array}{l}\text { FUNAYAMA; MOURA- } \\
\text { RIBEIRO; } \\
\text { GONÇALVES, } 1997 \\
\text { (Brasil) }\end{array}$ & Encefalopatia hipóxico-isquêmica. & Ensaio Cínico & 94 & $\begin{array}{l}\text { Dos } 15 \text { RNs com EHI grau II, que apresentaram convulsões neonatais, } 14 \text { foram } \\
\text { acompanhados por período de } 6 \text { anos (média de } 47 \text { meses). Houve reincidência } \\
\text { de crises em } 6 \text {, nos primeiros } 6 \text { meses. Destes, um com encefalopatia multifocal, } \\
\text { dois com síndrome de West e os demais com epilepsia focal. Analisando a fase } \\
\text { aguda da EHI dos casos que evoluíram com epilepsia e daqueles sem epilepsia, } \\
\text { constatou-se maior proporção de casos com epilepsia no subgrupo IIC ( } p=0,046) \text {. }\end{array}$ \\
\hline $\begin{array}{l}\text { BILLINGHURST et al, } \\
2017 \text { (EUA) }\end{array}$ & $\begin{array}{l}\text { Incidência e preditores de } \\
\text { epilepsia após acidente vascular } \\
\text { cerebral isquêmico arterial } \\
\text { pediátrico. }\end{array}$ & $\begin{array}{l}\text { Análise } \\
\text { retrospectiva }\end{array}$ & 218 & $\begin{array}{l}\text { Convulsões sintomáticas remotas (RSS) e epilepsia são importantes sequelas } \\
\text { neurológicas do AVC isquêmico Arterial (AIS) pediátrico. Crianças com acidente } \\
\text { vascular cerebral perinatal com convulsões sintomáticas agudas correm maior } \\
\text { risco desses desfechos. }\end{array}$ \\
\hline $\begin{array}{l}\text { PISANI et al., } 2017 \\
\text { (Itália) }\end{array}$ & $\begin{array}{l}\text { Período epileptogênico de } 15 \text { anos } \\
\text { após lesão cerebral perinatal. }\end{array}$ & Relato de caso & 1 & $\begin{array}{l}\text { Em conclusão, nosso relatório sugere que recém-nascidos com NS devido a um } \\
\text { acidente vascular cerebral perinatal têm um risco maior de epilepsia, com um } \\
\text { processo epileptogênico que pode durar mais de uma década. }\end{array}$ \\
\hline $\begin{array}{l}\text { ARAÚJO; } \\
\text { FONTENELLE; PIRES, } \\
2011 \\
\text { (Brasil) }\end{array}$ & $\begin{array}{l}\text { Síndrome de West: remissão } \\
\text { precoce não é garantia de resultado } \\
\text { final normal. }\end{array}$ & $\begin{array}{l}\text { Estudo } \\
\text { retrospectivo de } \\
\text { séries de casos }\end{array}$ & 37 & $\begin{array}{l}\text { Etiologia em lactentes com síndrome sintomática de West, História neonatal de } \\
\text { hipóxia (10/29) foi a etiologia mais frequente. }\end{array}$ \\
\hline $\begin{array}{l}\text { YILMAZ et al., } 2016 \\
\text { (Turquia) }\end{array}$ & $\begin{array}{l}\text { Avaliação de dez fatores } \\
\text { prognósticos que afetam o desfecho } \\
\text { da síndrome de West. }\end{array}$ & $\begin{array}{l}\text { Estudo } \\
\text { retrospectivo }\end{array}$ & 216 & $\begin{array}{l}\text { A classificação etiológica do grupo de estudo; Hipóxia (29\%), distúrbios metabólicos } \\
(11 \%) \text {, doenças infecciosas }(9 \%) \text { e desenvolvimento cerebral distúrbios alimentares } \\
(8 \%) \text { foram os mais frequentes fatores. }\end{array}$ \\
\hline
\end{tabular}

Fonte: Dados da pesquisa, 2018.

REAS/EJCH | Vol. Sup. 24 | e1136 | DOI: https://doi.org/10.25248/reas.e1136.2019 Página 4 de 6 


\section{DISCUSSÃO}

O presente estudo teve como objetivo definir se a hipóxia neonatal tinha relação ou não com a ocorrência se epilepsia. Entretanto houve algumas barreiras em relação a quantidade de estudos sobre o tema, pois os estudos relacionados a etiologia da epilepsia são escassos. Porém mesmo com as dificuldades pode se chegar a uma conclusão sobre a pergunta norteadora.

Pode se observar e outros autores relataram que o cérebro de uma criança no primeiro ano de vida é muito imaturo e a hipóxia de poucos minutos já pode provocar um dano enorme aos neurônios. Isso se dá porque ainda há uma imaturidade muito grande do sistema nervoso, o que os deixam mais propensos a adquirir doenças neurológicas (ARAÚJO APQC et al., 2011).

Nesse contexto, a Síndrome de West é um tipo de encefalopatia epiléptica que pode causar atraso no desenvolvimento cerebral de uma forma progressiva e evoluir para problemas psicomotores, e quanto mais demorado se iniciar o tratamento, maior será o número de crises e mais extenso será o dano cerebral (VELÁSQUEZ NH et al., 2014).

A epilepsia tem tratamento, que é realizado através do controle com antiepilépticos, existem vários tipos de drogas que normalmente são eficazes para o tratamento, só que nem sempre isso ocorre, além do mais algumas pessoas podem sofrer de reações indesejáveis. O ideal é que seja feito esse acompanhamento adequado e o primordial seria a prevenção da doença através de um parto e pré-natal adequado (FERNANDES MJS, 2013).

Em uma pesquisa realizada com registro de pacientes portadores de epilepsia com idade entre 3 e 44 anos, foram selecionados 18 registros de pacientes para realização da análise de dados, destes metade do sexo feminino e a outra metade do masculino. O estudo concluiu que metade dos pacientes com síndrome de Lennox-Gastaut, que também é um tipo de encefalopatia epiléptica da infância, tiveram parto prematuro ou ameaça de aborto, com isso pode se observar que fatores pré e perinatais influenciam no surgimento da síndrome. Além do mais pode se observar um atraso no desenvolvimento dessas crianças (YILMAZ S et al., 2016).

Alguns autores identificam a presença de um atraso neuropsicomotor em crianças com Encefalopatia Hipóxica Isquemica, verifica-se também a presença de outras doenças e fatores neurológicos, como a epilepsia e paralisia cerebral, além de outras sequelas (PISANI F et al., 2009). Diante do presente estudo pode se verificar que hipóxia neonatal é uma das causas da epilepsia.

\section{CONSIDERAÇÕES FINAIS}

Apesar do número limitado de estudos, que abordam o assunto pesquisado, encontrados na literatura foi possível observar que alguns fatores perinatais da epilepsia podem ser preveníeis, abrindo assim caminho para novas pesquisas onde deverá ser explorado todos os fatores possíveis da epilepsia, com o objetivo de criar estratégias de prevenção que possam ajudar a reduzir os índices de epilepsia infantil, com isso sugerimos novos estudos para que o tema possa ser aprofundado.

\section{REFERÊNCIAS}

1. ÁLVAREZ IV, MARTíNEZ PM. Caracterización etiológica del síndrome de Lennox-Gastaut sintomático. Revista Cubana de Pediatría, 2012; 84(1): 22-32.

2. ARAÚJO APQC, FONTENELLE LMC, PIRES L. West syndrome: early remission is not assurance of normal final outcome. Child Care Health Dev., 2011; 37(2): 261-65.

3. ARGUDIN XEM, DUARTE YD, GIL EP et al. Estudio Epidemiológico de la epilepsia infantil en el municipio de Bahía Honda. Rev. Ciências Médicas, 2011; 15 (1): 76-88.

4. BILLINGHURST LL, BESLOW LA, ABEND NS et al. Incidence and predictors of epilepsyafter pediatric arterial ischemic stroke. Neurology, 2017; 88(7): 630-37.

5. ECHÁVEZ FLF, TABARES CS, MILA RAS et al. Características clínicas y eletroencefalográficas de los pacientes con Síndrome de Lennox-Gastautenel programa de epilepsia de laUniversidad de Antioquia. Medellín20072012.ActaNeurológicaColombiana,2015;31(1): 2-11. 
6. FERNANDES MJS. Epilepsia do lobo temporal: mecanismos e perspectivas. Estudos Avançados, 2013; 27(77): 85-98.

7. FONSECA GS, MORORÓ DDS, MEDEIROS YKF etal.Epidemiologicalaspectsofrefractoryepilepsyinapediatricshospital. Journal of Nursing UFPE online, 2016; 10(3): 1466-73.

8. FUNAYAMA CAR, MOURA-RIBEIRO MVL, GONÇALVES AL. Encefalopatia hipóxico- isquêmica. Arq. Neuropsiquiatr., 1997 ; 55 (4): 771-79.

9. GALVÃO TF, PANSINI TSA, HARRAD D. Principais itens para relatar Revisões sistemáticas e Meta-análises: A recomendação PRISMA. Epidemiologia e Serviços de Saúde, Brasília, 2015; 24(2): 335-42.

10. GALVÃO TF, PEREIRA MG. Revisõessistemáticasdaliteratura:passosparasuaelaboração. Epidemiologia e Serviços de Saúde, $2014 ; 23(1): 183-84$.

11. KUMAR A, PALIWAL V K, AGARWAL V et al. Relationship of Lennox-Gastaut syndrome with perinatal event: A cross- sectional study. JournalofPediatricNeurosciences,2015;10(2):98-102.

12. MEDINA P. Síndrome de West, eldesafío de una atención oportuna. Revista de Neuropsiquiatria, $2017 ; 78$ (2): $63-64$.

13. MINAMIHARA CT, LIBERALESSO V, POSSAS SA et al. Perinatal brain lesions and epilepsy: Prematurity and cerebral hypoxia evolving with West Syndrome. Journal of Epilepsy and Clinical Neurophysiology, 2015; 21(4): 130-35.

14. PISANI F, ORSINI M, BRAIBANTI S et al. Development of epilepsy in newborns with moderate hypoxic-ischemic encephalopathyandneonatalseizures. Brain\&Development,2009;31(1):64-68.

15. PISANI F, PAVLIDIS E, FACINI C et al. A 15-year epileptogenic period after perinatal brain injury. FunctionalNeurology, 2017 ; 32 (1): 49-53.

16. VELÁSQUEZ NH, BORJAS IL, MATOS AA. Evaluación neurológica en recién nacidos con asfixia al nacer. Correo Científico Médico de Holguín, 2014; 18 (3): 457-68.

17. YILMAZ S, TEKGUL H, SERDAROGLU G et al. Evaluation of ten prognostic factors affecting the outcome of West syndrome. Acta Neurol Belg., 2016; 116(4): 519-27. 